This document is SAE-copyrighted intellectual property. It may not be shared, downloaded, duplicated, reprinted, or transmitted in any manner without prior written permission from SAE. SAE requires that you make best efforts to secure and protect the document from disclosure, taking at least the same care that you would for your own confidential information.

\title{
Thank you. \\ The Safety and Dynamic Performance of Blended Brake System on a Two-Speed DCT Based Battery Electric Vehicle
}

\author{
Jiageng Ruan, Paul Walker, Nong Zhang, and Guangzhong Xu \\ University of Technology Sydney
}

\begin{abstract}
Regenerative braking has been widely accepted as a feasible option to extend the mileage of electric vehicles (EVs) by recapturing the vehicle's kinetic energy instead of dissipating it as heat during braking. The regenerative braking force provided by a generator is applied to the wheels in an entirely different manner compared to the traditional hydraulic-friction brake system. Drag torque and efficiency loss may be generated by transmitting the braking force from the motor, axles, differential and, specifically in this paper, a two-speed dual clutch transmission (DCT) to wheels. Additionally, motors in most battery EVs (BEVs) and hybrid electric vehicle (HEVs) are only connected to front or rear axle. Consequently, conventional hydraulic brake system is still necessary, but dynamic and supplement to motor brake, to meet particular brake requirement and keep vehicle stable and steerable during braking. Therefore, a complicated effect on the safety and performance of braking, mainly relating to tyre slips and locks, vehicle body bounces and braking distance will be applied by the blended brake system.
\end{abstract}

In this paper, the brake energy recovery potentials of typical driving cycles are presented. Relevant critical limitations are introduced to define the available brake force distribution range for front and rear axles. Then the distribution strategies are compared and analyzed to achieve a satisfied balance between braking performance, driving comfort and energy recovery rate. Next, the required motor brake force is tuned, according to the response time and efficiency loss in transfer process which obtained in testing bench. At last, solutions for some special cases are proposed, for instance, motor brake torque interruption when downshifting occurs on long downhill.

A credible conclusion is gained, through experimental validation of optimized brake force distribution strategy on a two-speed DCT based BEV testing rig, that the selected force distribution strategy help the blended brake system achieve a comfortable and safety braking during all driving conditions.

CITATION: Ruan, J., Walker, P., Zhang, N., and Xu, G., "The Safety and Dynamic Performance of Blended Brake System on a Two-Speed DCT Based Battery Electric Vehicle," SAE Int. J. Passeng. Cars - Mech. Syst. 9(1):2016, doi:10.4271/2016-01-0468.

\section{INTRODUCTION}

Due to the great potential of reusable braking energy in daily driving, the blended brake system, which consists of electric and hydraulic brake, has been validated theoretically and experimentally to be effective on a battery electric vehicle (BEV) by a plethora of papers $[\underline{1}, \underline{2}, \underline{3}, \underline{4}]$. Most of these researching mainly focus on the improvement of braking energy rate, nevertheless, lose the sight of safety issue that new blended brake system brings. Even the few papers focused on the performance of controllability and stability for a blended brake system, the massive strong brake required situations and the balance of braking performance and kinetic energy recovery ability are still out of scope $[\underline{5}, \underline{6}]$.

More and more BEVs utilize a multi-speed transmission, e.g. Automatic Transmission (AT), Automated Manual Transmission (AMT) and Continuously Variable Transmission (CVT), to improve vehicle dynamic performance and extend one-charge driving range $[\underline{7}, \underline{8}, \underline{9}]$. An advanced and simplified two-speed Dual Clutch

Transmission (DCT) has been proven to be more efficient, at the same time, help vehicle recapture more braking energy $[\underline{10}, \underline{11}, \underline{12}]$. However, this new architecture of powertrain introduces more uncertainty to the blended brake system and needs some special designed algorithms to ensure a safety braking, in the meanwhile, recovery energy from braking as much as possible. The safetyoriented control algorithm applied on the conventional vehicles, e.g. Anti-lock Brake System (ABS), Electro Stability Program (ESP), should also be integrated to the blended brake system after some reasonable modifying $[\underline{13}, \underline{14}]$. Therefore, a completed strategy, which considers the braking performance, reliability, durability, cooperating with gearbox, interacting with driver assistance system (ABS, ESP, etc.), is badly needed.

The aim of this paper is to study, evaluate and finally supply a reliable and effective strategy for blended braking system in two speeds DCT based EV. A manually selected braking strategy is proposed in this paper to achieve the balance of driving experience and energy recovery ability, which comes along with automatically intervened top-priority control strategy. First of all, kinetic energy 
recovery potential is calculated to evaluate the researching work for a more complex regenerative braking strategy. Then, individual pros and cons introduced by the two-speed DCT based front drive BEV, for instance, load transferred to motor connected axle and 'torque hold' in shifting, is discussed. Three different strategies are designed to recapture more braking energy, achieve the best brake performance and compromise between performance and energy. Next, a Matlab/ Simulink ${ }^{\circledR}$ model is established to analyze the details of brake force distribution, wheel slip and kinetic energy recovery. Finally, strategies are tested in the electrified powertrain testing bench.

\section{KINETIC ENERGY RECOVERY POTENTIAL}

Braking energy recovery is regarded as one of the greatest improvement, which can help save money and energy, instead of wasted by heat in traditional Internal Combustion Engine (ICE) vehicle. However, due to the completely different working principles and routes, some potential risk will be introduced to vehicles.

Therefore, more complicated strategies and equipment are needed. It is important to know how much energy is consumed by braking and if it is worth that spending extra money on additional equipment and R\&D to achieve a satisfied brake system for BEVs. Fig.1 clearly shows the theoretical energy consumed by braking in several typical driving cycles. For city cycles or hybrid cycles, like LA92

Dynamometer Driving Schedule (LA92, Unified Driving Schedule), New Europe Driving Cycle (NEDC) or Urban Driving Dynamometer Schedules (UDDS), the energy wasted in braking accounts for more than $40 \%$. Even in highway cycle, i.e. Highway Fuel Economy Testing (HWFET), relative fewer acceleration and deceleration, it takes over $20 \%$. Actually, the energy wasted in braking can easily go over $50 \%$ during daily peak time commuting driving, especially in metropolises. Even not all of them can be recaptured; RBS can significantly extend driving range, in other words, save your money.

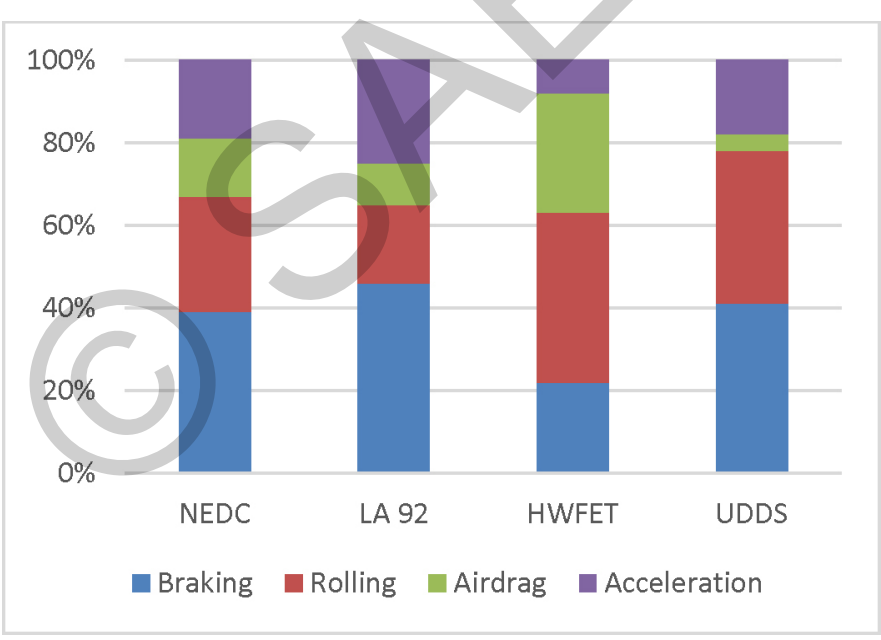

Figure 1. Energy Consumption Distribution in Driving Cycles

\section{Vehicle Specifications}

The vehicle parameters used in this studying are shown in Table.1:
Table 1. Vehicle Specifications

\begin{tabular}{|c|c|c|c|}
\hline Parameters & Symbol & Value & Unit \\
\hline Battery Capacity & $\mathrm{C}$ & 72 & $\mathrm{Ah}$ \\
\hline Motor Torque (Peak/Rate) & $\mathrm{T}_{\text {peak }} / \mathrm{T}_{\text {rate }}$ & $300 / 150$ & $\mathrm{Nm}$ \\
\hline Motor Power (Peak/Rate) & $\mathrm{P}_{\text {peak }} / \mathrm{P}_{\text {rate }}$ & $125 / 45$ & $\mathrm{Kw}$ \\
\hline Transmission Ratio & $\mathrm{i}_{1} / \mathrm{i}_{2}$ & $2.15 / 1.46$ & \\
\hline Main Reducer & $\mathrm{i}_{\mathrm{g}}$ & 3.93 & \\
\hline Centre of Mass Height (CoM) & & 0.5 & $\mathrm{~m}$ \\
\hline CoM to Front Wheel & & 1.3 & $\mathrm{~m}$ \\
\hline CoM to Rear Wheel & & 1.5 & $\mathrm{~m}$ \\
\hline Vehicle Mass & $\mathrm{m}$ & 1850 & $\mathrm{Kg}$ \\
\hline Tyre Radius & $\mathrm{r}$ & 0.3125 & $\mathrm{~m}$ \\
\hline Air Drag Coefficient & $\mathrm{C}_{\mathrm{D}}$ & 0.28 & \\
\hline Road Friction Coefficient & $\mu$ & 0.9 & \\
\hline Vehicle Front Area & $\mathrm{A}$ & 2.2 & $\mathrm{~m}^{2}$ \\
\hline
\end{tabular}

\section{POWERTRAIN TOPOLOGY}

The advantages and details of two-speed DCT based BEV has been introduced [15]. In this paper, only braking relevant topics, which caused by this new DCT structure, are studied. Fig. 2 is a schematic diagram that shows the principle of two-speed DCT based powertrain, on the top left, and the structure of testing bench.

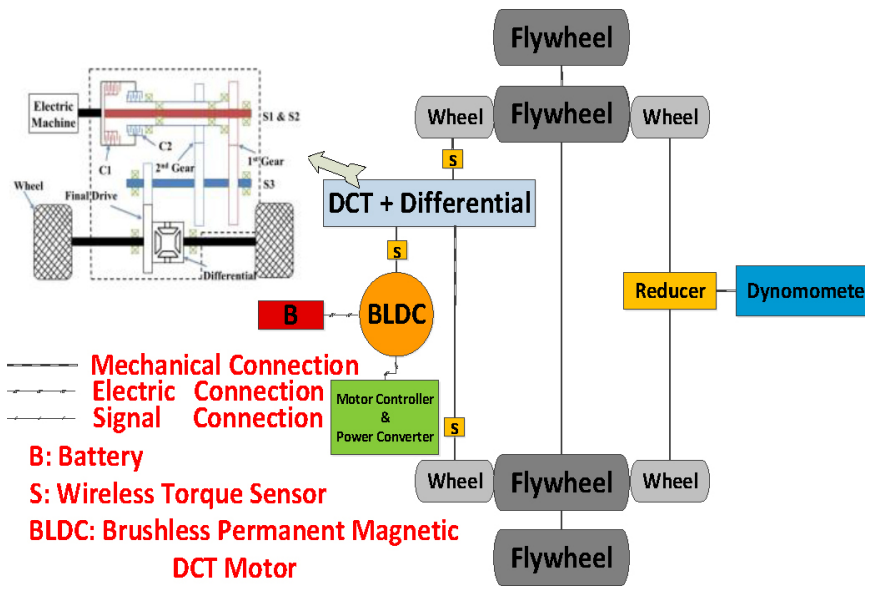

Figure 2. Two-Speed DCT based BEV Powertrain Structure

The front drive BEVs has lots of common merits same to the traditional ICE vehicles, such as cheaper and easier to build, a more capacious room. Furthermore, this structure provides an extra benefit for regenerative braking dcue to the load trancsfer in BEVs.

Load transfer is presented when vehicle is accelerating or decelerating, no matter it theoretically supposed to be a rigid vehicle or a vehicle has suspension system that would exhibit pitching or rolling of the body. The effect of pitching and rolling only adds small load transfer [16], in this paper, the vehicle is assumed to be a perfectly rigid body without suspension, considering the main target is safety and energy optimization. The dynamic adding weight during braking are expressed as Eq.1: 


$$
\Delta \text { Weight }_{\text {front }}=\mathrm{a} \frac{\mathrm{h}_{\mathrm{g}}}{\mathrm{W}} \mathrm{m}
$$

a is the longitudinal deceleration, $h_{g}$ is the height of mass center, $\mathrm{w}$ represents the wheelbase length and $\mathrm{m}$ stands for the vehicle mass. The ratio do normal force distributed on front and rear wheels at different deceleration are illustrated in Fig.3. Comparing to the static state, $1 \mathrm{~g}$ deceleration will transfer almost $40 \%$ load from rear wheels to front ones. As shown in Eq.2, the normal load of wheel decides the maximum available friction torque on a certain road. Which means the limitation of wheel unlocked friction ability will increase during braking. And the regenerative brake from front-mounted motor can play a greater role in braking and energy recovery.

$$
\mathrm{F}_{\mathrm{b}}=\mu \mathrm{F}_{\mathrm{R}}
$$

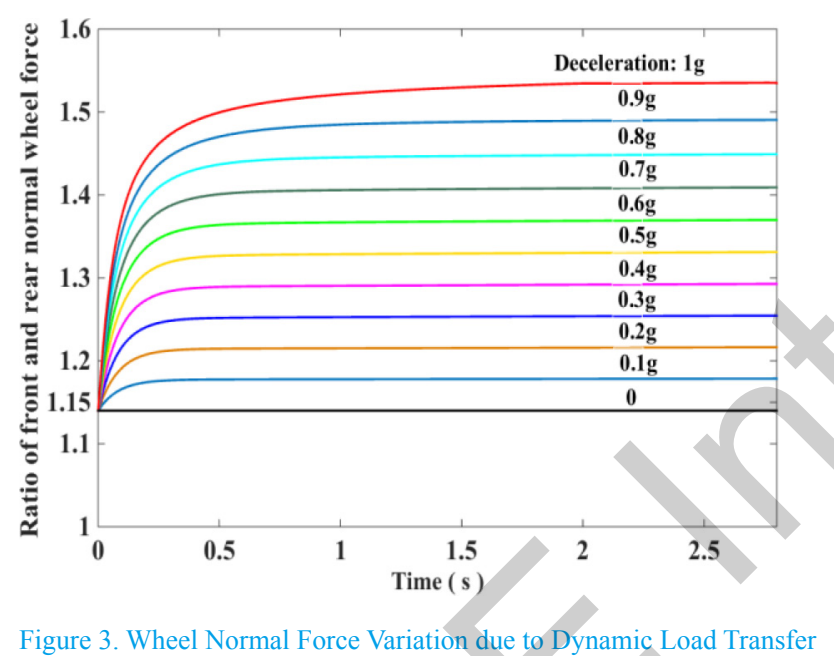

Figure 3. Wheel Normal Force Variation due to Dynamic Load Transfer

\section{SAFETY AND STABILITY DESIGN IN BLENDED BRAKE SYSTEM}

The goal of automotive brake system design, no matter for conventional vehicle or BEVs, is to achieve a comfortable and reliable decelerating as the request of driver. And stop vehicle as soon as possible in emergency situation, meanwhile, maintaining dynamic stability and controllability.

On the longitudinal direction, the relative motion between tyre and road will turn from a mix of sliding and rolling to pure sliding, if the force applied on the wheel by caliper exceeds the maximum available friction force between tyres and ground (Eq.3). This phenomenon is also known as 'wheel lock'.

$$
f_{\text {caliper }}>f_{\text {brake_friction }}=m g \mu
$$

$\mu$ is friction factor depending on road conditions and type of tyre. The motion of wheel in a normal driving vehicle consists of two parts, i.e. rolling and sliding, which causes a difference between the speeds of vehicle and wheel. The wheel slip ratios is defined as the difference between the rotational speed of the wheel and the translational velocity of the wheel center:

$$
\lambda=\frac{\Delta v}{v}=\frac{\omega r_{d y n}-v}{v}
$$

$\omega$ is wheel rotation speed; $r_{d y n}$ is the dynamic radius of wheel, which is achieved indirectly by measuring the travel distance per rotation circle. $\lambda$ is a value from 0 to $100 \%$ representing the motion of wheel from freely rolling to lock. The solid blue curve in Fig. 4 shows friction factor $(\mu)$ varying according to longitudinal slip ratio $(\lambda)$ on dry asphalt pavement. The $\mu$ drops significantly when vehicle riding on the wet and snow road, which are presented by solid and green dash curve in Fig.4. Moreover, a steering angle causes the friction factor to fall as well.

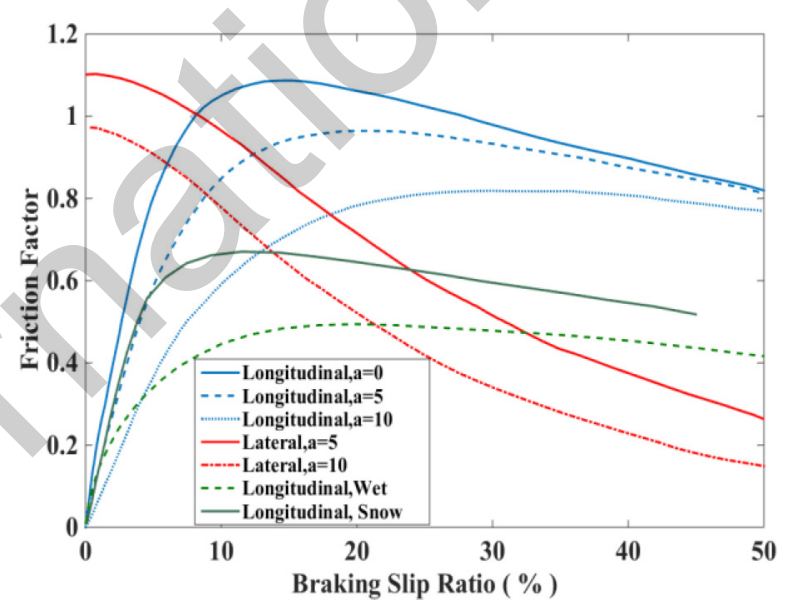

Figure 4. The Influence of Slip Ratio, Steering Angle and Road Condition on Friction Factor

The force on lateral direction of road-tyre contact surface directly affects the direction control ability of vehicle. Locked wheel cannot generate lateral force to offset the sideslip trend, when cornering or unintentional steering during emergency brake, resulting in unnecessary under-steering and uncontrollable over-steering. As shown in Fig.4, the lateral friction factor falls dramatically with longitudinal braking slip ratio increasing. Take the example of a wheel with $5^{\circ}$ steering angle and $20 \%$ longitudinal slip ratio, the lateral friction factor only equals half of pure straight driving. When the longitudinal slip ratio goes up to $100 \%$ (Wheel lock), steering input has no result on yaw motion because the front tyres are saturated, and no lateral force can be generated. If it happens to the front wheel, the vehicle lost steering ability, however, there is no directional instability because "whenever the lateral movement of the front wheels occurs, a self-correcting moment due to the inertial force of the vehicle about the yaw center of the rear axle will be developed. Consequently, it tends to bring the vehicle back to a straight line path. On the contrast, if rear wheels are locked, they lose their capability to generate the required side forces and the rear end might start to slide sideways, losing directional stability. The omitted arrows on rear wheel and front wheels, in the 'Over-steering diagram' and 'Understeering diagram' respectively of Fig.5, indicate the locked wheels and lost lateral force. 

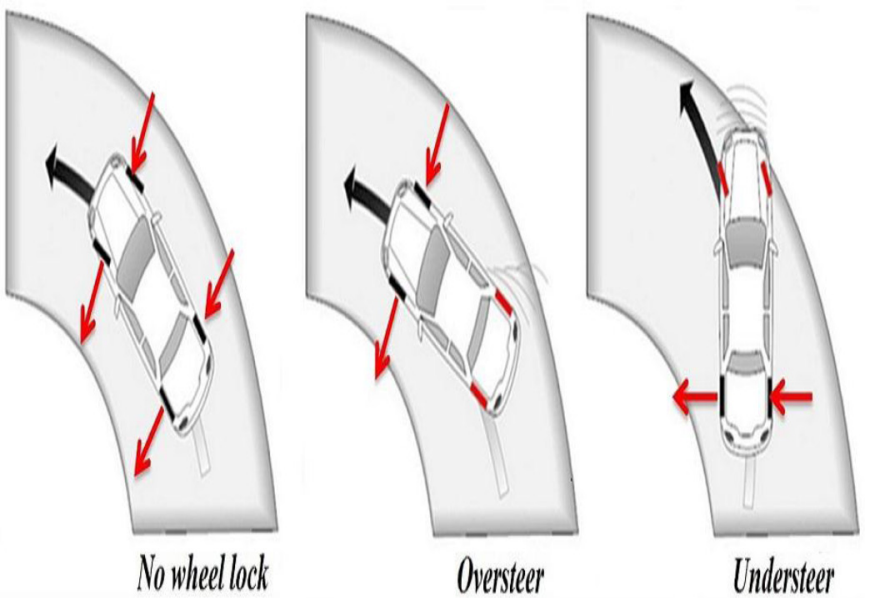

Figure 5. Over-steering and Under-steering when Wheels Lock

In traditional ICE vehicles, to ensure maximum brake force available and avoid slipping, lots of components and strategies are introduced into vehicle, e.g. Anti-lock Brake System (ABS), Electronic Stability Program (ESP), Electronic Brake Force Distribution (EBD) and Brake Assistance System (BAS). All the above functions rely on hydraulic actuators and work together. In brief, EBD supply appropriate force to help ESP effectively keep vehicle running at initial intended path, meanwhile, ABS ready to prevent any wheel lock. However, in the RBS, the strategies and intervention time of hydraulic brake system are changed, aiming at braking energy recovery.

When the deceleration intention is detected from brake pedal, motor begins to provide brake torque on front wheels; meanwhile, pressure is established in the rear hydraulic actuator to decelerate rear wheels. The force distribution between front and rear wheels is decided by Eco strategy, which aims at recovering energy as much as possible if the brake intention is moderate. EBD distribute more brake force on the front wheel to offset the load transfer, shown in Fig.3, and avoid rear wheels locking. When vehicle is loaded with passengers or goods, EBD detects it and redistributes more brake force on rear wheels to utilize the increased available friction force. (Shown in Fig.6) The variation of force on front and rear wheels, which is usually supplied by hydraulic system, can be provided by motor at the viewpoint of energy recovery. The brake force on front vehicle is provided by motor until it exceeds the rotation speed based maximum available torque, then, hydraulic-mechanical brake supplies the rest of required torque (Fig.6-1). When sensors detect added load, the previous distribution strategy is kept at the viewpoint of energy recovery, instead of increasing rear brake force and reducing front brake force immediately, at the cost of a longer stop distance (Fig.6-2B). However, this only happens in moderate braking $(a<$ $0.3 \mathrm{~g}$ ). Stop distance becomes the top concern when detected braking intention going stronger $(a>0.3 g)$. Brake force distribution is rebalanced to take full advantage of load transfer. Rear mechanical brake force is increased, at the same time, reduce front mechanical brake and keep motor brake, or reduce motor brake if no mechanical brake on front wheels. The detail procedures are described in the flowchart (Fig.7).

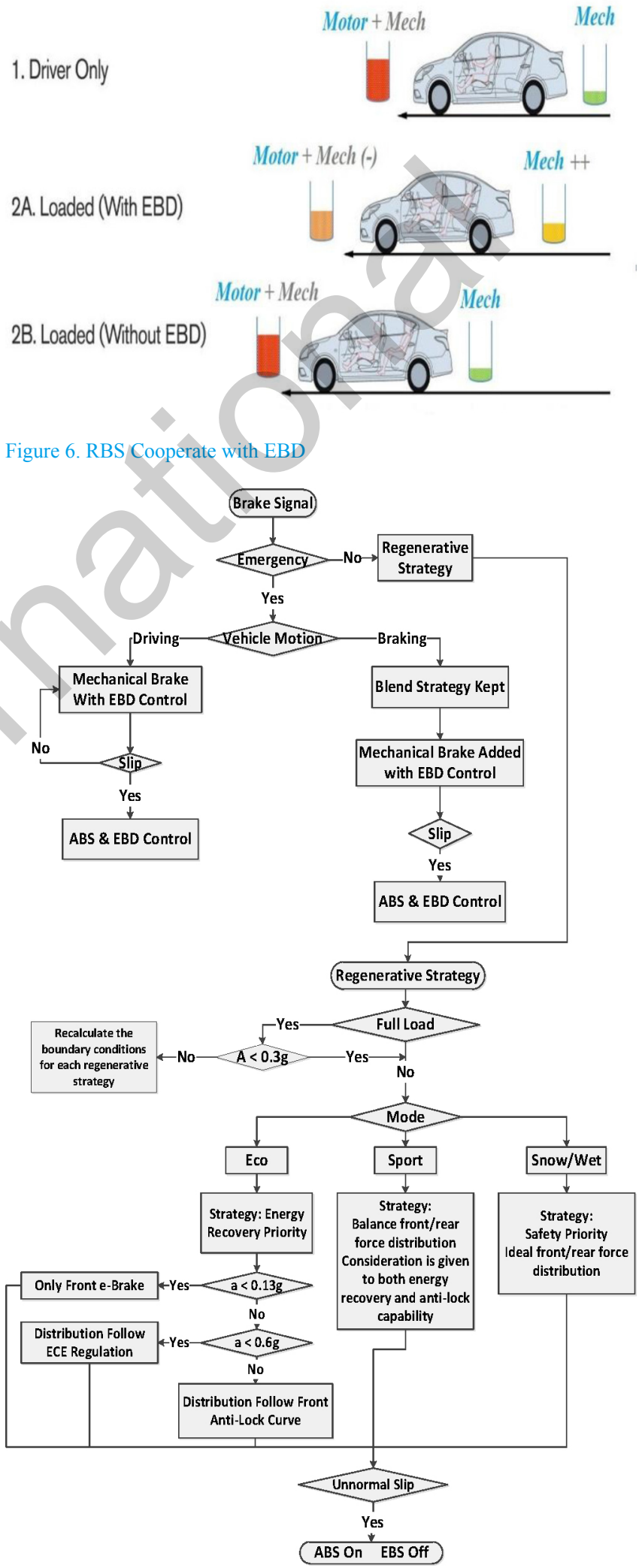

Figure 7. The Cooperation of RBS, EBD and ABS

ABS gets involved in EBD when emergency brake is activated. ABS reduces the pressure in the hydraulic brake actuator of the wheel that tending to lock. However, there are two different preconditions for the blended brake system when ABS works: 
1. Emergency brake starts from driving situation

2. Increasing brake starts from moderate braking situation

In case 1, emergency brake usually needs a great deal of force. For RBS, it can generate high instantaneous current in the motor, which can't be taken by battery. And considering HBS has a higher reliability, hydraulic ABS is given the highest priority, which means motor brake does not participate in emergency brake in this situation.

In case 2 , there is already some level of regenerative brake before the brake strength turning to strong. Removing the existed generative brake and refill mechanical brake to reach required level not only wastes time but also introduces possibility of insufficient brake torque during translation. Both of them are fatal during an emergency situation even for a short moment. The strategy proposed in this paper, in this case, keeps regenerative brake unchanged and the rest required torque is supplied by HBS with ABS assistance. Fig. 6 shows how the mechanical and electrical brake works jointly during emergency case.

Another advantage of electric brake comparing to traditional mechanical one is the durability and high-temperature resistance. No matter the materials selected for brake disk and pad, the wear, deformation and even failure at high temperature are evitable, sometimes fatal. Motor electric brake eliminates all these potential risk by directly applying negative torque on rotating shafts.

\section{REGENERATIVE BRAKE CAPABILITY}

The available regenerative brake on front wheels is restricted by motor characteristics, speed and gear ratio. As we can see from Fig.8, maximum brake force from motor is limited under $5000 \mathrm{~N}$ and continues dropping when vehicle runs in $2^{\text {nd }}$ gear. Even vehicle runs in $1^{\text {st }}$ gear with a bigger torque amplification ratio, and it can only supply $8000 \mathrm{~N}$, not to mention these maximum torques only available in starting period until each gear's 'turning point', shown in Eqn.5. It is another reason why mechanical brake system still necessary for BEVs, except the safety concern.

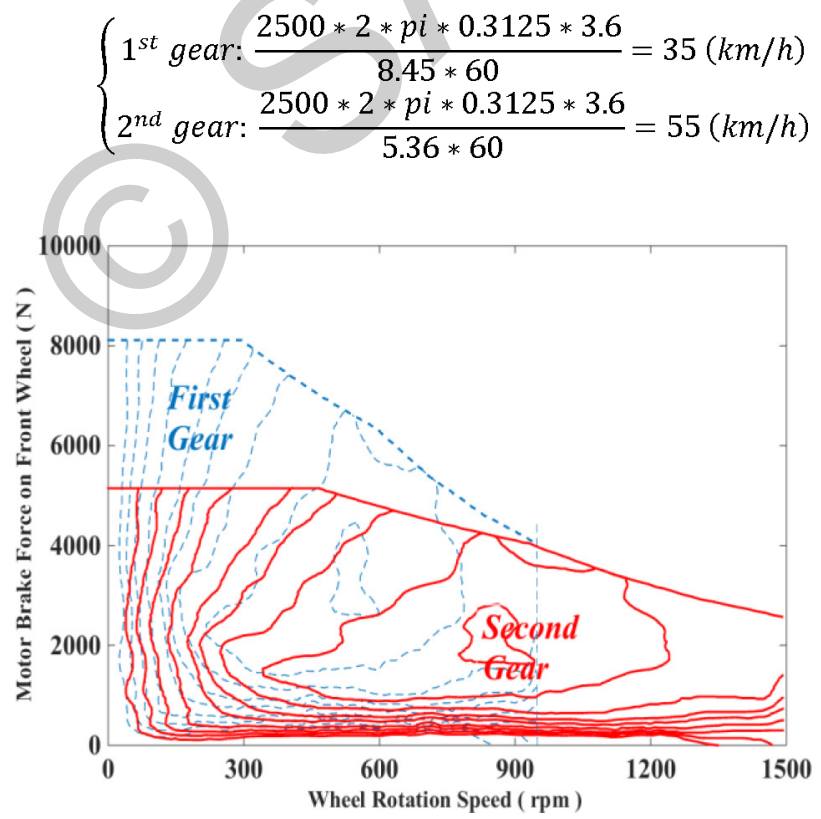

Figure 8. Available motor brake force in different gears and motor efficiency

\section{BRAKING STRATEGIES}

The ideal strategy properly distributes brake force to each wheel, which goes through all the intersection of friction factors based front and rear friction limitations, to ensure the maximum use of road friction force and all the wheels lock simultaneously. Of course, any wheel lock will be avoided by ABS.

Except the ideal brake force distribution strategy, other strategies result in asynchronous wheels locking time, then, possible oversteering or under-steering. However, wheel-lock only comes along with emergency brake or extreme road conditions, which can be compensated by functions like ABS, EBD and ESP. Considering the regenerative brake supplier-front axle connected motor, reducing front brake torque wastes the reusable kinetic energy potential for most daily commuting, which only requires mild and moderate braking. Therefore, it is necessary to design some strategies to recover braking energy as much as possible before any intervention of safe oriented strategy.

According to Eqn.3, friction factor is the determinant of maximum force wheel, which can be generated before wheel-lock. Generally, $\mu$ is less than 1.2 , which means deceleration is lower than $1.2 \mathrm{~g}(\mathrm{~g}=9.8$ $\mathrm{m} / \mathrm{s}^{2}$ ). However, the deceleration can go over $3 \mathrm{~g}$ by improving vehicle aerodynamics and special designed road, e.g. Formula 1. In this paper, considering the various road conditions and tyres types used by ordinary passenger vehicle, which determine friction factor jointly, $\mu$ is set to 0.9 for safety at the cost of wasting some braking capability. The dotted lines in Fig.9 represent the maximum available force based on different friction factors $\mu$ and vehicle specifications in Table.1.

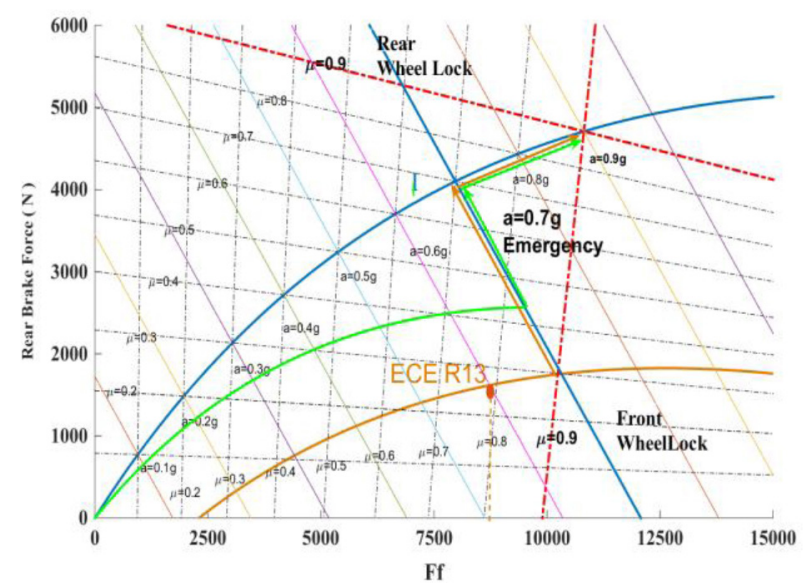

Figure 9. Braking Force Distribution on Front and Rear Wheels

The maximum decelerations in different driving cycles are presented in Table 2. The highest one, $2.2\left(\mathrm{~m} / \mathrm{s}^{2}\right) / 9.8\left(\mathrm{~m} / \mathrm{s}^{2}\right)=0.22 \mathrm{~g}$ appeared in LA-92 cycle, is far away from the wheel-lock deceleration thresholds, represented by two red dotted curves in Fig.9 respectively.

Table 2. Max Deceleration in Typical Driving Cycles

\begin{tabular}{|c|c|c|c|c|c|}
\hline & NEDC & UDDS & JP-1015 & HWFET & La 92 \\
\hline Max Deceleration $\left(\mathrm{m} / \mathrm{s}^{2}\right)$ & -1 & -0.92 & -0.66 & -1.4 & -2.2 \\
\hline
\end{tabular}


Referring to the previous analysis of safety and stability issue during braking, for all load states of vehicle, the adhesion utilization curve of the rear axle must not lie above the curve for the front axle. Reflected in the Fig.9, it means the distribution curve should be always under ideal strategy curve (I). Also, law in most countries very closely controls brake system across the world to ensure that road vehicles are designed and constructed to brake safely and efficiently under all conditions of operation. There are lots of brake related regulations and directives from worldwide governments and organizations. The European UN Regulations 13 is recognized as valid type approval standards in all EU and lots of un-EU countries, e.g. members of the agreement 1958 as Japan, USA, Canada, Australia, Korea, China, India and Malaysia. It requires that for all states of loading of two-axle motor vehicles that are not equipped with $\mathrm{ABS}$, the rate of braking must meet the requirement of $3^{\text {rd }}$ equation in Eqn.6:

$$
\left\{\begin{array}{c}
F_{b f}=\mu m g\left(\frac{L_{b}+z h_{g}}{L}\right) \\
F_{b r}=\mu m g\left(\frac{L_{a}-z h_{g}}{L}\right) \\
z=\frac{a}{g}>0.1+0.85(\mu-0.2)
\end{array}\right.
$$

$F_{b f}$ and $F_{b r}$ are the dynamic maximum friction force on front and rear wheels during decelerating caused load transfer. $L_{a}$ and $L_{b}$ are the distance from wheel centre to CoM. Then, the corresponding relationship of front and rear brake force under ECE R13 regulations is achieved and shown in golden curve in Fig.9 and Eqn.7:

$$
\left\{\begin{array}{c}
F_{b f}=\frac{z+0.07}{0.85} \frac{G}{L}\left(L_{b}+z h_{g}\right) \\
F_{b r}=m g z-F_{b f}
\end{array}\right.
$$

Like the function of driving mode selection in lots of sport and luxury ICE vehicles, the brake strategies are ready to set by driver manually in this BEV blended brake system.

\section{Snow/Wet/Emergency Strategy}

The braking safety issue, e.g. stopping distance, stability and controllability, is always the top priority and likely to be tested by bad weather/road conditions and emergency brake. Therefore, if the strategy is manually set to snow/wet, or deceleration rate goes over threshold value- $0.7 \mathrm{~g}$, the brake force will be ideally distributed to front and rear wheels, i.e. blue curve I in Fig.9, to make the maximum utilization of road-tyre friction force. When vehicle closes to stop in emergency brake, the deceleration is lower than the emergency situation threshold. Strategy may be altered from emergency to Eco automatically, which is unnecessary, especially in a short time. Thus, strategy alternation is locked during emergency brake until stop. The details of this strategy are referred to Fig.7 and cases $1 \& 2$ in page 4 .

\section{Eco Strategy}

To maximize the recovery potential of braking energy, only front electric brake is available before deceleration rate reaches the intersection point, which is determined by horizontal axis and ECE R13 regulation curve. After that, the ratio of front and rear axle brake force follows the ECE regulation curve until the deceleration trigger the emergency situation-a $0.7 \mathrm{~g}$ decelerating. Then, the distribution strategy jumps to 'Ideal' I curve.

\section{Sport Strategy}

Aggressive driving is common when driver intentionally selects this strategy. Heave acceleration and deceleration, especially more frequent start-stop, may increase the error possibility of motor. Besides, it gives the $2^{\text {nd }}$ gear of DCT more chance to participate, in which the relative smaller ratio restricts the motor brake torque. The maximum electric brake in $2^{\text {nd }}$ gear is:

$$
\text { Brake }_{2 n d}=\frac{T_{p e a k} \cdot g_{2}}{r}=\frac{300 \times 5.36}{0.3125}=5146(N)
$$

Mechanical brake has to compensate the insufficient regenerative brake frequently to achieve satisfied stopping distance. Comparing to the Eco strategy that electric brake has priority and mechanical brake works as a supplement, a 'parallel' strategy is more appropriate in this case. 'Parallel' means hydraulic-mechanical system and motor supply brake torque jointly with specific ratio on the front wheels from very beginning. Refer to Fig.3, the front normal load is about 1.15 times of the rear normal load and the ratio increases to 1.35 when a $0.8 \mathrm{~g}$ emergency brake occurs. To ensure front wheels lock before rear wheels do, the brake force distribution ratio should always be higher than this value. The mechanical brake force distribution ratio follows the ideal strategy (I curve), in the meanwhile, additional motor brake is added to front wheels, which is equals to $20 \%$ of front mechanical brake. This 'Sport' strategy, green curve in Fig.9, keeps the vehicle stable and controllable at any deceleration rate and can supply ideal brake force distribution even in motor brake faulty, of course, at the cost of lower braking energy recovery rate.

\section{Motor Fault Insurance Strategy}

Generally, electromagnetic equipment is considered to be not as robust as a hydraulic system. Specific to the brake system, motor downtime is a very dangerous situation, no matter caused by IGBT failure or temperature protection. Especially in long downhill braking, continuously generating big current by braking may cause overheating issue, then, trigger protection mechanism when insufficient cooling or the cooling system is out of order. It is not a common, but serious, event. Hydraulic brake fail-safe provision should be activated immediately when brake torque is 'limited' or 'torque error' situation is detected. Fig.10 demonstrates a fail-safe mechanism of motor, given consideration to motor overload ability and error redundancy. 


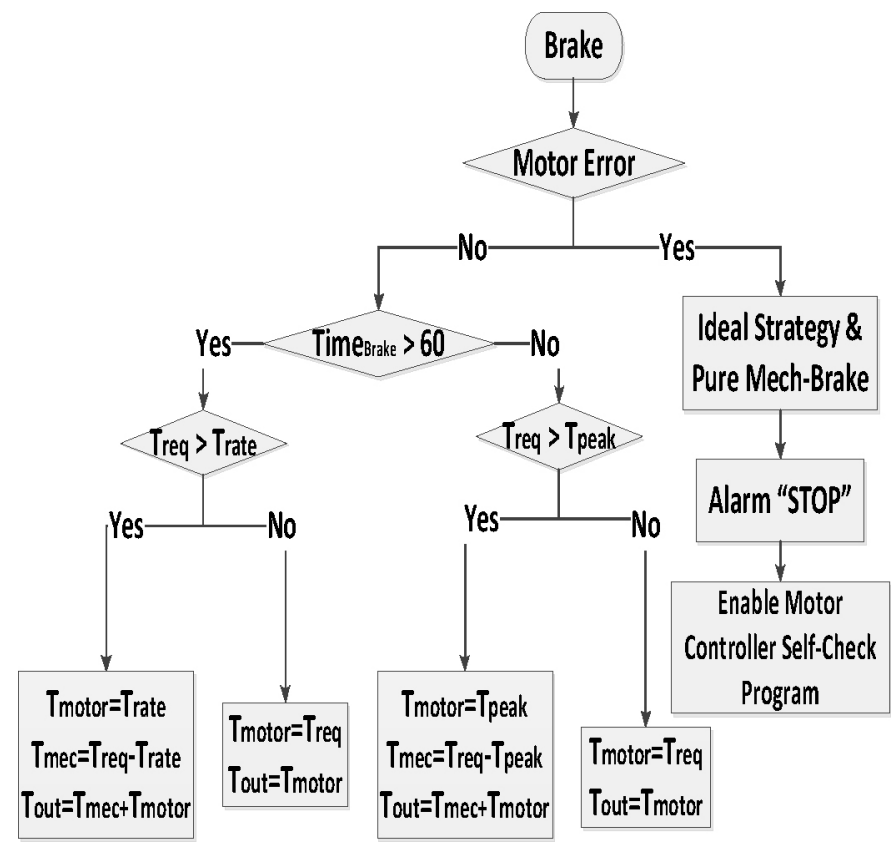

Figure 10. Motor Control \& Fail-Safe Strategy

\section{Braking Strategy during Shifting}

The motor regenerative brake and mechanical-hydraulic brake have entirely different working path to decelerate vehicle. Electric brake goes through transmissions and differentials, then, acts on the half shafts, which are connected to each wheel. The mechanical brake goes from brake pedal to master cylinder, hydraulic actuator and calipers, then, directly acts on the wheels. One side, the regenerative brake from motor may be insufficient when vehicle running at high speed with smaller gear ratio. On the other side, the torque interruption introduced by gear shifting can lead to serious potential safety issue, especially for emergency brake. Although, the shifting time in DCT is very short, the interruption shown in Fig.11, also known as 'shifting torque hole', still can be felt, which may send the wrong message to drives and cause some unnecessary correct measure. There are two possible solutions:

1. Lock shifting functions and uses mechanical brake to supply the rest of required.

2. Use mechanical brake to supply the reduced torque during braking, and then, taken over by brake torque from $1^{\text {st }}$ gear after shifting.

Obviously, the second solution can recapture more braking energy by giving regenerative brake more opportunities to take part in. However, it also needs a more complicate control algorithm and higher requirement for the cooperation of mechanical and electric brake. Considering the occurrence frequency, cost, potential recovery energy from emergency brake during a short time and safety risk, solution 1 is the favored choice for market products.

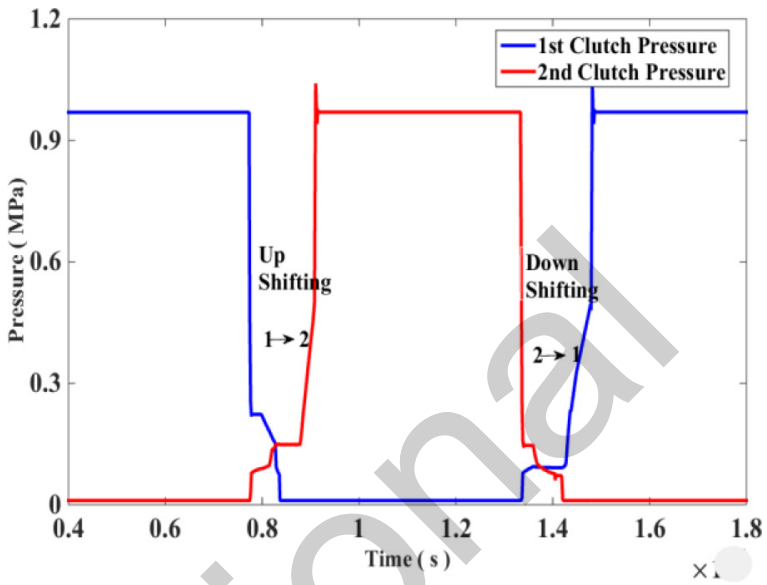

Figure 11. Clutch Pressure Varying during Braking

\section{SIMULATION ANALYSIS}

The simulation model used in this study, which is designed for the assessment of the safety and energy recovery performance of braking, is a backward-facing model shown in Fig.12. Vehicle control unit (VCU) calculates the required driven and braking torque from motor, friction brake from hydraulic system and power from battery, starting from the velocity profile of the assigned driving schedule. Then, the torque and power are limited and distributed by component characteristics and selected strategies. Next, electric torque, through transmission system, cooperates with mechanical torque, which is applied directly, working on the wheels.

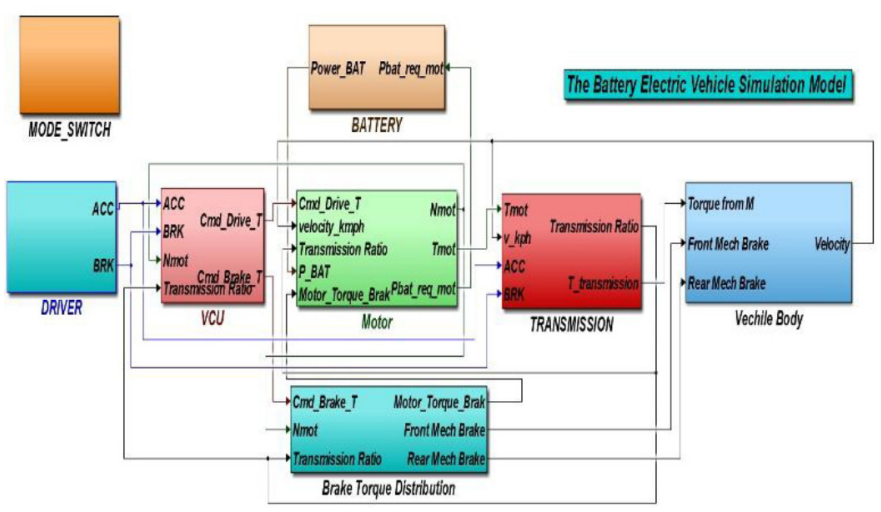

Figure 12. Two-Speed DCT based BEV Simulink $®$ Model

Braking performance of BEV with three different strategies is simulated in the NEDC, UDDS, HWFET, LA92, and JP1015 (Japan City Cycle). They stand for typical moderate and aggressive city driving and highway cruising.

\section{Braking in Typical Cycles}

The brake force applied on the front and rear wheels, in different strategies, i.e. Eco, Sport and Ideal, are shown in Fig.13, taking the example of NEDC. The vehicle is decelerated to stop as required, no matter which strategy is selected, presented in Fig.14. In additional to the amount difference of required brake force, the brake force distribution curves (ratios) are similar in other cycles. 


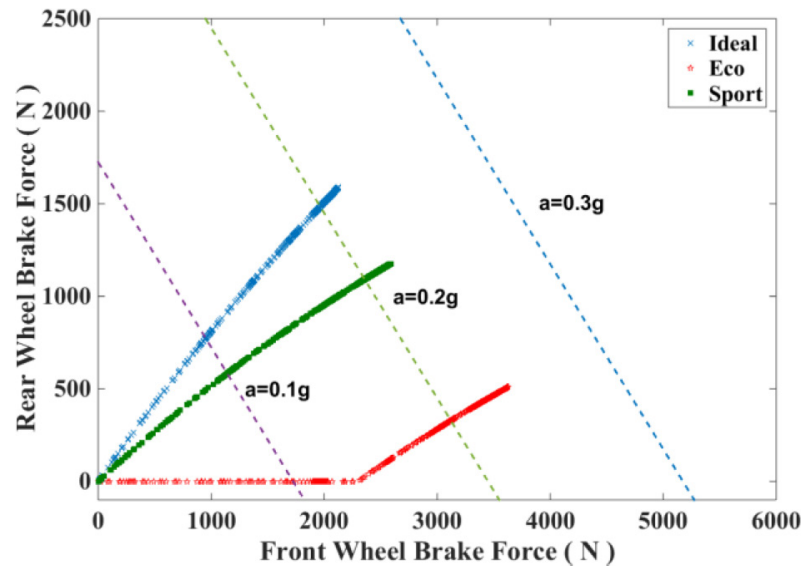

Figure 13. Ratio of front/rear brake force in different strategies

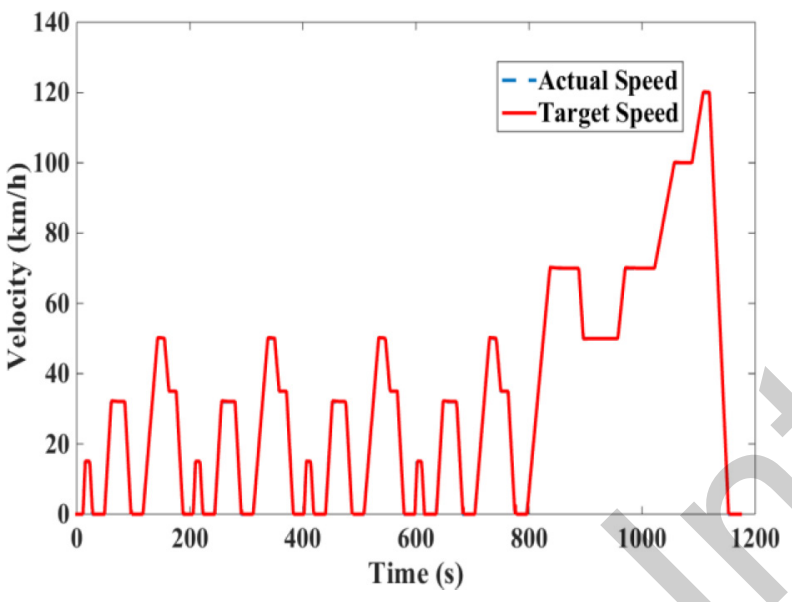

Figure 14. Vehicle Speed and Speed Profile

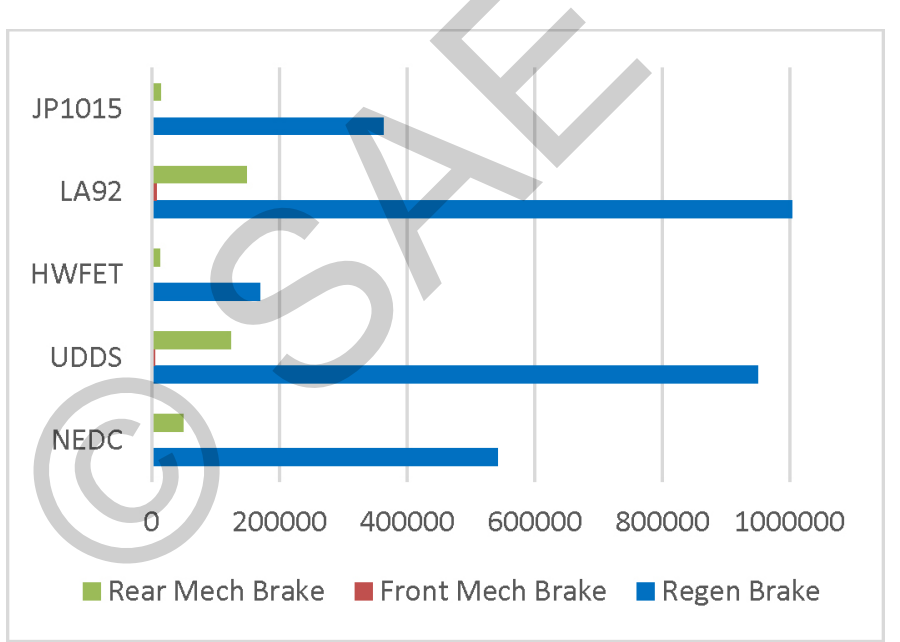

Fig. 15. Braking Force Distribution for Eco Strategy in Driving Cycles

The maximum deceleration in each typical cycle, as shown in Table.2, is far smaller than the $0.7 \mathrm{~g}$ emergency brake threshold. Therefore, the ideal brake force distribution strategy will not be activated in cycles, except the Snow/Wet mode is manually selected. Fig. $15 \& \underline{16}$ show the details of brake force distribution for Eco and Sport strategies in various driving cycles. With the help of a strong motor and a big battery, most of the required brake torque is supplied by motor only in Eco strategy in all testing cycles. Rear wheel mechanical brake will participate by the requirement of ECE R13regulation when deceleration goes higher. Mechanical brake force on front wheels has little chance to play a role in stopping the vehicle in this strategy.

The situation in Sport strategy is different. The brake force on front wheels is compulsively distributed between mechanical and electric system at a fixed ratio. Regenerative brake is restricted aiming at insure safety brake all the time.

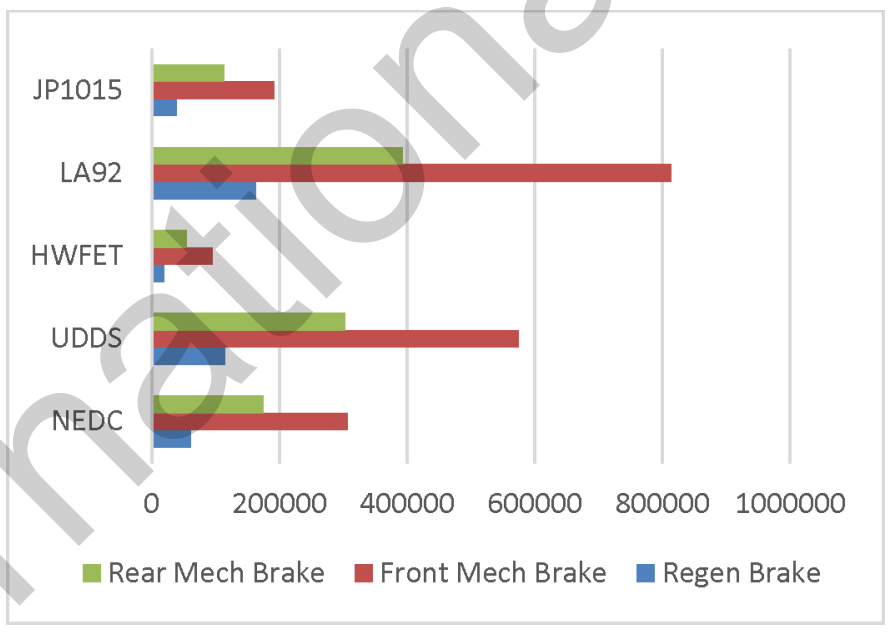

Figure 16. Braking Force Distribution for Sport Strategy in Driving Cycles

The braking energy recovery rate is used in this study, expressed by Eq.9, to evaluate strategies, except safety performance. The rate is defined as the ratio of battery input energy from braking and battery output energy for driving:

$$
Q_{r e}=\frac{B a t_{I N}}{B a t_{O U T}} \times 100 \%
$$

Table 3 presents the comparison of braking energy recovery rate in strategies and cycles. Aggressive city driving can recapture more energy than others, e.g. UDDS and LA92. The reason why JP1015 is one of the cycles have high recovery rate is the required driving energy is not much bigger, comparing to the input energy from braking. Most of them does not reach the threshold of ECE regulation, which means required brake is supplied by motor only for most time. Due to the fewer required brake force comparing to driving force, the recovery rate of HWFET is the lowest one. Safetyoritened Sport strategy results in much lower energy recovery rate. None of them goes over $10 \%$ in all cycles.

Table 3. Energy Recovery Rate

\begin{tabular}{|c|c|c|c|c|c|}
\hline Energy Recovery Rate (\%) & NEDC & UDDS & JP-1015 & HWFET & LA92 \\
\hline Eco Strategy & $40.4 \%$ & $58.8 \%$ & $59.9 \%$ & $21.6 \%$ & $61.2 \%$ \\
\hline Ideal Strategy & 0 & 0 & 0 & 0 & 0 \\
\hline Sport Strategy & $6.1 \%$ & $7.8 \%$ & $8.0 \%$ & $3.1 \%$ & $8.9 \%$ \\
\hline
\end{tabular}




\section{Braking in Emergency}

Referring to the 'Safety and Stability' charter, there are two different emergency brake cases. Fig. 17 shows the brake force on front and rear wheels when a strong brake applies on a driving vehicle. Front brake force is kept larger than the rear one, according to the rules in Ideal strategy, after both of them responses fast to braking pedal signal.

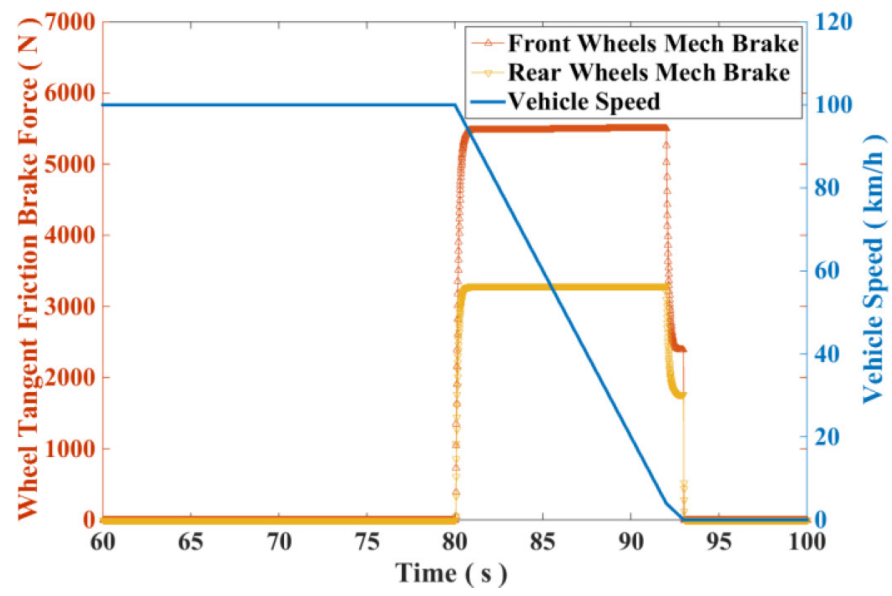

Figure 17. Emergency brake occurs during driving

Fig.18 demonstrates the varying of brake force distribution when emergency brake applies on a braking vehicle. At the initial period, a moderate brake results in the selection of Eco strategy. Regenerative brake accounts for most of required, in the meanwhile, a small amount mechanical brake applies on rear wheels to keep vehicle controllable and stability. The strategy, then, is automatically changed to 'Ideal' when brake pedal goes deeper. Regenerative brake from motor is kept after strategy alternation and mechanical brake acts on wheels very fast to play a leading role in this situation.

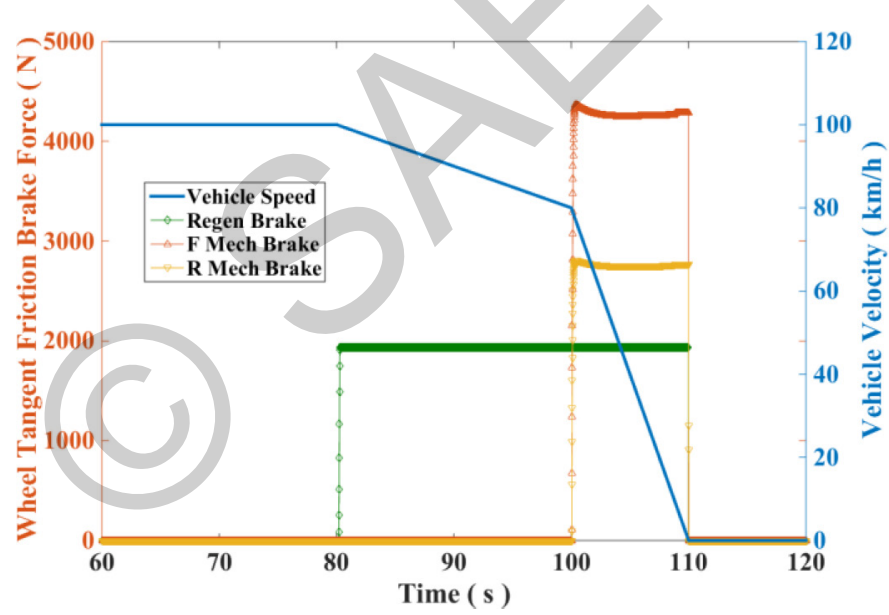

Figure 18. Emergency brake occurs during braking

Fig.19 illustrates the slip ratios of front and rear wheels during braking. With the help of ABS and EBD, slip ratios are kept in a high-efficiency area to make the most use of friction force from road, according to Fig. 4

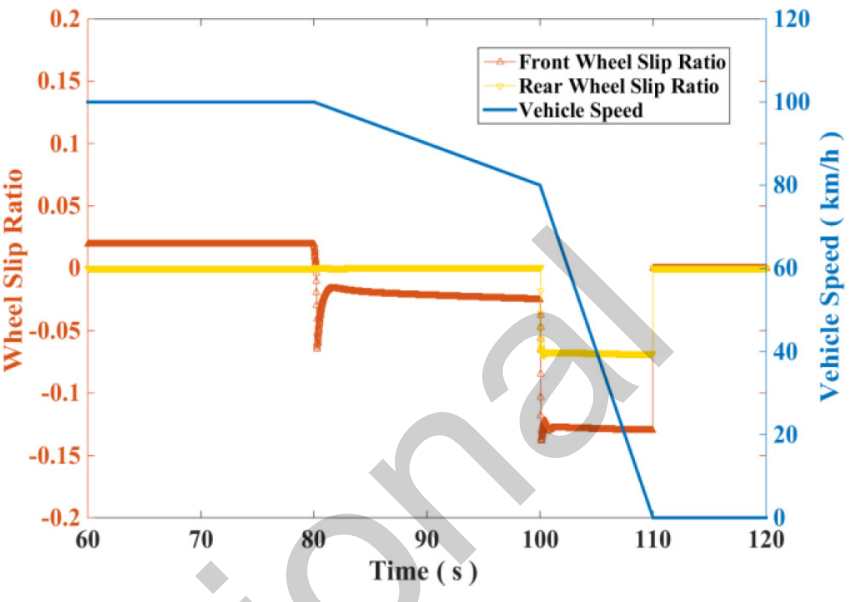

Figure 19. Wheels slip ratios in case 2 Emergency braking

\section{EXPERIMENTAL RESULTS}

The powertrain-testing bench consists of BLDC motor, dSPACE ${ }^{\circledR}$ prototyping ECU, differential integrated two speeds DCT, wheels, flywheels, dynamometer and pneumatic-hydraulic mechanical brake system, as shown in Fig. 20 \& 21 . Four flywheels are designed to simulate the inertia of a vehicle with a mass of $1850 \mathrm{~kg}$.

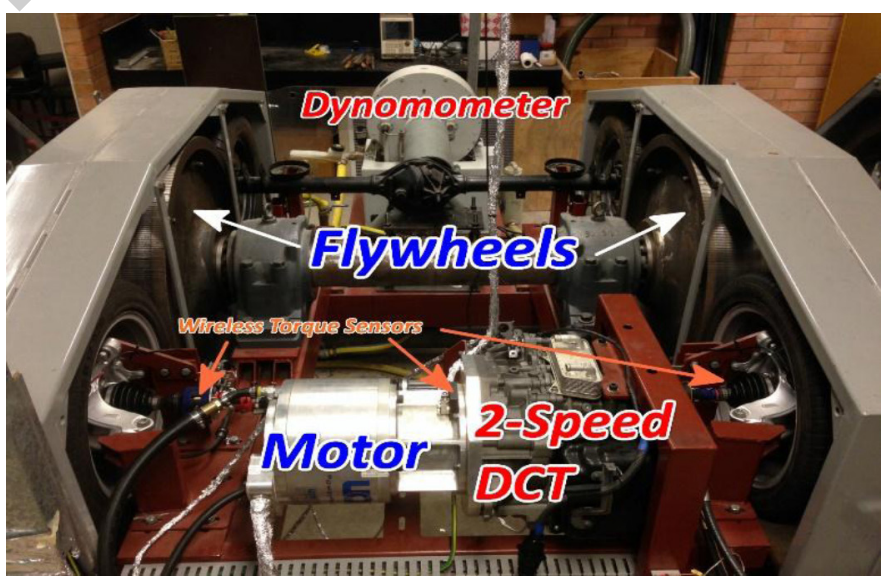

Figure 20. Powertrain Testing Bench

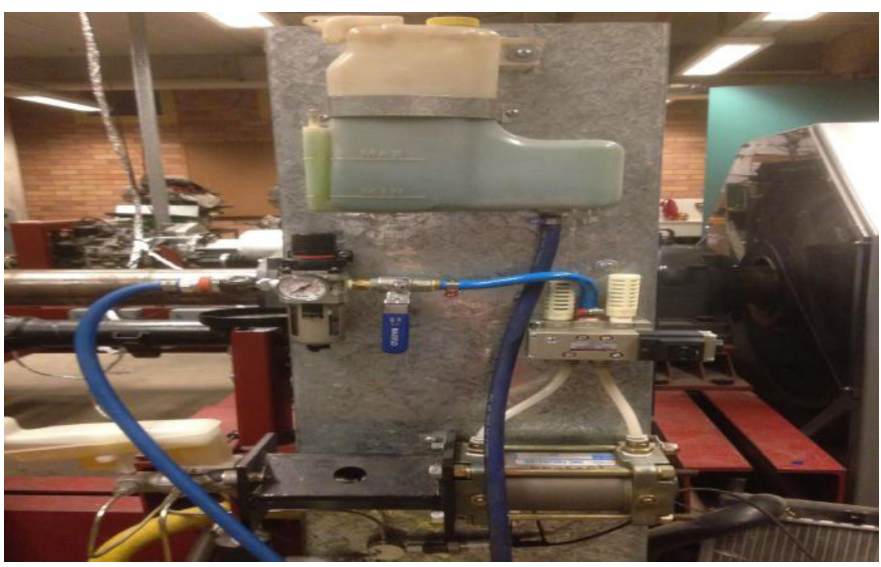

Figure 21. Pneumatic-Hydraulic Mechanical Brake System 
This testing rig was designed to analysis powertrain performance through a half-vehicle model. The pneumatic-hydraulic brake system can only decelerate front wheels. Therefore, the corresponding rear mechanical brake is supplied by dynamometer as request. Fig. 22 \& 23 demonstrate the brake force distribution in NEDC for 'Sport' strategy. The blended brake system decelerates the flywheels accurately according to the requirement of speed profile.

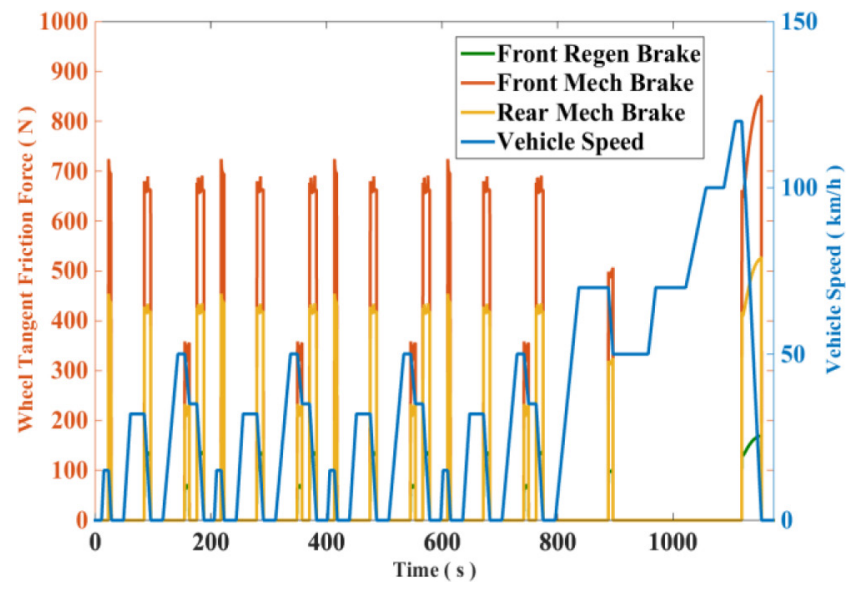

Figure 22. Sport Strategy Brake Force Distribution in NEDC

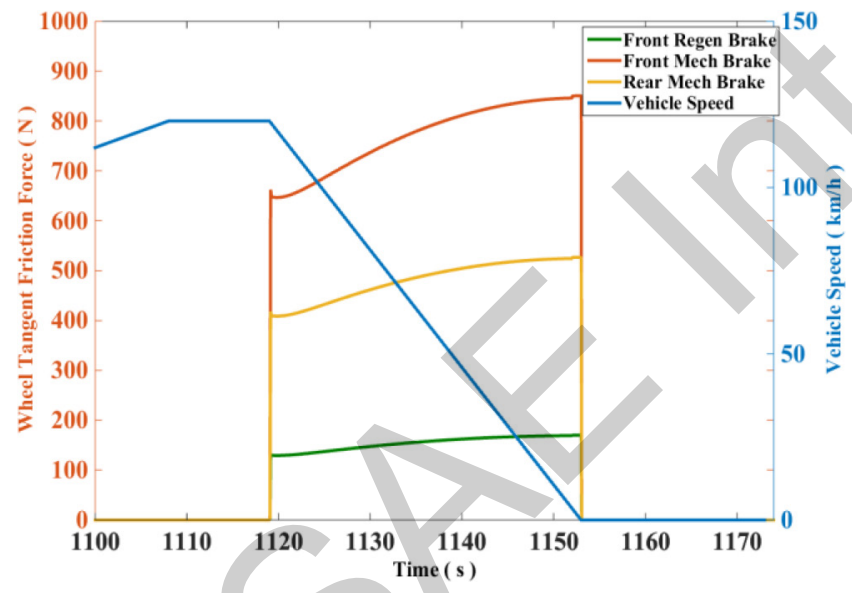

Figure 23. Detail View of Sport Strategy Brake Force Distribution in NEDC

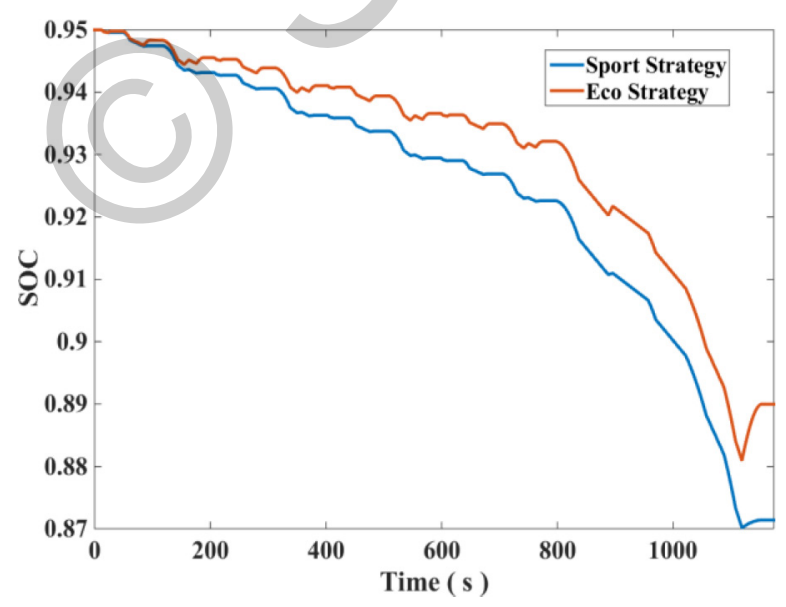

Figure 24. SOC varying in Eco and Sport Strategy in NEDC
Fig.24 indicates the varying of battery State of Charge (SOC) in 'Eco' and 'Sport' strategies in one NEDC. 'Eco' strategy has a significant advantage of 'Sport' strategy in energy saving. The SOC of 'Eco' strategy has a bigger increase, comparing to that of 'Sport', in every braking period. At the end of cycle, a 15\% improvement is recorded.

\section{SUMMARY}

This paper proposes three blended brake system working strategies, aiming at energy recovery, safety driving and bad road condition/ emergency situation respectively, after showing the great kinetic energy recovery potential in daily driving cycles. The possible safety issues, which may be caused by redistributed brake force between front/rear axles and mechanical/regenerative brake system, are analyzed and eliminated by special designed algorithms. The potential brake torque interruption risk introduced by shifting in two-speed DCT is avoided by proposed strategies as well. An integrated BEV model, established in Matlab/Simulink ${ }^{\circledR}$, clearly demonstrates kinetic energy recovery rate, wheel slip ratios and the difference of braking force distributions in various cycles, by different regenerative braking strategies. The experimental verified results prove that the special designed strategies are effective and reliable for the two-speed DCT based blended brake system.

\section{REFERENCES}

1. Lv, C., Zhang, J., Li, Y., and Yuan, Y., "Regenerative Braking Control Algorithm for an Electrified Vehicle Equipped with a By-Wire Brake System," SAE Technical Paper 2014-01-1791, 2014, doi:10.4271/201401-1791.

2. Hartley, J., Day, A., Campean, I., McLellan, R. et al., "Braking System for a Full Electric Vehicle with Regenerative Braking," SAE Technical Paper 2010-01-1680, 2010, doi:10.4271/2010-01-1680.

3. Kubaisi, R., Herold, K., Gauterin, F., and Giessler, M., "Regenerative Braking Systems for Electric Driven Vehicles: Potential Analysis and Concept of an Adaptive System," SAE Technical Paper 2013-01-2065, 2013, doi:10.4271/2013-01-2065.

4. Nadeau J, Boisvert M, Micheau P. Implementation of a Cooperative Strategy between a Vehicle's Mechanical and Regenerative Brake System. Veh Power Propuls Conf (VPPC), 2014 IEEE 2014:1-6. doi:10.1109/VPPC.2014.7007086.

5. Zhang, J., Lv, C., Yue, X., Qiu, M. et al., "Development of the Electrically-Controlled Regenerative Braking System for Electrified Passenger Vehicle," SAE Technical Paper 2013-01-1463, 2013, doi:10.4271/2013-01-1463.

6. Crolla DA, Cao D. The impact of hybrid and electric powertrains on vehicle dynamics, control systems and energy regeneration. Veh Syst Dyn 2012;50:95-109. doi:10.1080/00423114.2012.676651.

7. Jun-Qiang X, Guang-Ming X, Yan Z. Application of automatic manual transmission technology in pure electric bus. 2008 IEEE Veh Power Propuls Conf VPPC 2008 2008:5-8. doi:10.1109/VPPC.2008.4677583.

8. Bottiglione F, De Pinto S, Mantriota G, Sorniotti A. Energy Consumption of a Battery Electric Vehicle with Infinitely Variable Transmission. Energies 2014;7:8317-37. doi:10.3390/en7128317.

9. Di Nicola, F., Sorniotti, A., Holdstock, T., Viotto, F. et al., "Optimization of a Multiple-Speed Transmission for Downsizing the Motor of a Fully Electric Vehicle," SAE Int. J. Alt. Power. 1(1):134-143, 2012, doi:10.4271/2012-01-0630.

10. Walker PD, Abdul Rahman S, Zhu B, Zhang N., Modelling simulations, and optimisation of electric vehicles for analysis of transmission ratio selection. Adv Mech Eng 2013;2013. doi:10.1155/2013/340435.

11. Zhu B, Zhang N, Walker P, Zhan W, Zhou X, Ruan J. Two-speed DCT electric powertrain shifting control and rig testing. Adv Mech Eng 2013;2013:323917-1 - 323917-10. doi:10.1155/2013/323917.

12. Ruan, J. and Walker, P., "An Optimal Regenerative Braking Energy Recovery System for Two-Speed Dual Clutch Transmission-Based Electric Vehicles," SAE Technical Paper 2014-01-1740, 2014, doi: $\underline{10.4271 / 2014-01-1740 .}$. 
13. Infantini, M., Britto, J., and Perondi, E., "Model of an ABS Pneumatic Regenerative Braking System," SAE Technical Paper 2005-01-4033, 2005, doi:10.4271/2005-01-4033.

14. Conlon B, Kidston K. Electric vehicle with regenerative and anti-lock braking, 1997.

15. Walker PD, Zhang N. Modelling of dual clutch transmission equipped powertrains for shift transient simulations. Mech Mach Theory 2013;60:47-59. doi:10.1016/j.mechmachtheory.2012.09.007.

16. Gritt PS. Energy Conversion Management. 2011.

\section{CONTACT INFORMATION}

Jiageng Ruan

Mobil: +61 0450580627

RUANJIAGENG@GMAIL.COM

Mail Address: Unit T02, 4-12 Garfield St, Five Dock 2046, AUSTRALIA

\section{ACKNOWLEDGMENTS}

This researching work is supported by Powertrain Lab, Faculty of Engineer and IT, University of Technology Sydney. During this period, Prof. Nong Zhang, Dr. Paul Walker gave me a lot of valuable suggestions and comments. I am really appreciating the contribution they did for this studying.

\section{DEFINITIONS/ABBREVIATIONS}

BEV - Battery Electric Vehicle

HEV - Hybrid Electric vehicle

DCT - Dual Clutch Transmission

AT - Automatic Transmission

AMT - Automated Manual Transmission

CVT - Continuously Variable Transmission

VCU - Vehicle Control Unit

ABS - Anti-Lock Brake System

ESP - Electro Stability Program

EBD - Electro Control Brake Distribution

BAS - Brake Assistance System

RBS - Regenerative Brake System

SOC - State of Charge

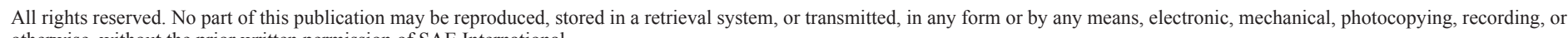
otherwise, without the prior written permission of SAE International. 